Foxp $3^{+}$and suppressive but that express no or low levels of CD25 (17). Additionally, one may consider whether Foxp3 in the human as opposed to the mouse is also expressed by activated $T$ cells, independently of any regulatory function.

The second concern, closely linked to the first, is the critical issue of the antigen specificity of the Tregs studied, which is only very indirectly addressed (14). The results showing a biased $\mathrm{T}$ cell repertoire restricted to a given $V \beta$ family ( $V \beta 2$ in this case) in a representative individual with persistent CMV infection are intriguing. However, the regulatory functional capacity of the $\mathrm{CD}^{+} \mathrm{CD} 25^{\text {hiFoxp }}{ }^{+}$cell subset detected in this $\mathrm{V} \beta 2$ anti-CMV population remains to be demonstrated.

To conclude, it appears plausible to extend to the human the dichotomy proposed in the mouse that distinguishes natural versus adaptive Tregs, which have distinct origins, namely, thymic-derived $\mathrm{CD} 4^{+} \mathrm{CD} 25^{+} \mathrm{Foxp}^{+}$cells in the case of natural regulatory lymphocytes versus peripheral memory-type $\mathrm{CD} 4^{+} \mathrm{CD} 25^{-}$precursors in the case of adaptive Tregs. In this context, it will be important to further experimentally dissect the adaptive Treg subset to more directly address whether or not the differences that have been proposed for each subset (e.g., Th2, Th3, Tr1, CD45RB low $\mathrm{T}$ cells) in terms of phenotype and putative cytokine dependency are indeed a reflection of their belonging to distinct cell lineages.

Address correspondence to: Jean-François Bach, INSERM U580, Hôpital NeckerEnfants Malades, 161 Rue de Sèvres, 75015 Paris, France. Phone: 33-144-49-53-73; Fax: 33-143-06-23-88; E-mail: bach@necker.fr.

1. Hori, S., Takahashi, T., and Sakaguchi, S. 2003. Control of autoimmunity by naturally arising regulatory CD4+ T cells. Adv. Immunol. 81:331-371.

2. Bach, J.-F. 2003. Regulatory T cells under scrutiny. Nat. Rev. Immunol. 3:189-198.

3. Chatenoud, L., Salomon, B., and Bluestone, J.A. 2001. Suppressor T cells-they're back and critical for regulation of autoimmunity! Immunol. Rev. 182:149-163

4. Bluestone, J.A., and Abbas, A.K. 2003. Natural versus adaptive regulatory T cells. Nat. Rev. Immunol. 3:253-257.

5. Sakaguchi, S. 2005. Naturally arising Foxp3expressing $\mathrm{CD} 25+\mathrm{CD} 4+$ regulatory $\mathrm{T}$ cells in immunological tolerance to self and non-self. Nat. Immunol. 6:345-352.

6. Waldmann, H., et al. 2006. Regulatory T cells in transplantation. Semin. Immunol. 18:111-119.

7. Coombes, J.L., Robinson, N.J., Maloy, K.J., Uhlig, H.H., and Powrie, F. 2005. Regulatory T cells and intestinal homeostasis. Immunol. Rev. 204:184-194. 8. Groux, H., et al. 1997. A CD4+ T-cell subset inhibits antigen-specific T-cell responses and prevents colitis. Nature. 389:737-742.

9. Roncarolo, M.G., and Levings, M.K. 2000. The role of different subsets of T regulatory cells in controlling autoimmunity. Curr. Opin. Immunol. 12:676-683.

10. Levings, M.K., et al. 2002. Human CD25+CD4+ $\mathrm{T}$ suppressor cell clones produce transforming growth factor beta, but not interleukin 10 , and are distinct from type $1 \mathrm{~T}$ regulatory cells. J. Exp. Med. 196:1335-1346.

11. Weiner, H.L., et al. 1994. Oral tolerance: immunologic mechanisms and treatment of animal and human organ-specific autoimmune diseases by oral administration of autoantigens. Annu. Rev. Immunol. 12:809-837.

12. Ochi, H., et al. 2006. Oral CD3-specific antibody suppresses autoimmune encephalomyelitis by inducing CD4(+)CD25(-)LAP(+) T cells. Nat. Med. 12:627-635.

13. Tisch, R., Wang, B., Atkinson, M.A., Serreze, D.V., and Friedline, R. 2001. A glutamic acid decarboxylase 65 -specific Th2 cell clone immunoregulates autoimmune diabetes in nonobese diabetic mice. J. Immunol. 166:6925-6936.

14. Vukmanovic-Stejic, M., et al. 2006. Human $\mathrm{CD} 4^{+} \mathrm{CD} 25^{\text {hi }} \mathrm{Foxp}^{+}$regulatory $\mathrm{T}$ cells are derived by rapid turnover of memory populations in vivo. J. Clin. Invest. 116:2423-2433. doi:10.1172/JCI28941.

15. Chen, W., et al. 2003. Conversion of peripheral CD4+CD25- naive $\mathrm{T}$ cells to $\mathrm{CD} 4+\mathrm{CD} 25+$ regulatory $\mathrm{T}$ cells by TGF-beta induction of transcription factor Foxp3. J. Exp. Med. 198:1875-1886.

16. Cobbold, S.P., et al. 2004. Induction of foxP3+ regulatory $\mathrm{T}$ cells in the periphery of $\mathrm{T}$ cell receptor transgenic mice tolerized to transplants. J. Immunol. 172:6003-6010.

17. Liu, W., et al. 2006. CD127 expression inversely correlates with FoxP3 and suppressive function of human CD4(+) T reg cells. J. Exp. Med. 203:1701-1711.

\title{
You say estren, I say estrogen. Let's call the whole replacement off!
}

\author{
Ushma S. Neill
}

Journal of Clinical Investigation, Columbia University College of Physicians and Surgeons, New York, New York, USA.

\begin{abstract}
Estrogens and androgens play a key role in regulating bone mass. However, their clinical use as bone anabolic agents is limited due to unwanted side effects, particularly in reproductive organs. In 2002, the synthetic ligand estren was described to reproduce the bone anabolic, nongenotropic effects of sex steroids while having no effect on the uterus or seminal vesicles. But in the current issue of the JCI, Windahl et al. provide data showing that estrens are not as suitable a replacement for estrogen as was initially reported (see the related article beginning on page 2500). Though not catabolic, estrens triggered only minor, nonsignificant increases in bone mass in gonadectomized mice, all the while inducing hypertrophy of reproductive organs. Does this mean estrens should not be pursued as a therapy for osteoporosis?
\end{abstract}

Nonstandard abbreviations used: AR, androgen receptor; ER, estrogen receptor; SERM, selective estrogen receptor modulator.

Conflict of interest: The author has declared that no conflict of interest exists.

Citation for this article: J. Clin. Invest. 116:2327-2329 (2006). doi:10.1172/JCI29733.

\section{Estrogen and its receptors}

The estrogen hormone family plays an essential role in the regulation of skeletal growth and homeostasis. While osteoblasts, osteocytes, and osteoclasts can be indirect targets of hormone signaling, they are also direct targets of estrogen and express functional estrogen and androgen receptors (ER and AR, respectively) (1). As estrogen or androgen deficiency can lead to rapid decreases in bone mass, therapies designed to return these sex hormones to their original levels would seem logical. However, these strategies have been fraught with difficulty due to the complex nature of hormone signaling.

In the classical (genomic) model of estrogen signaling, estrogens bind to the ER in the nucleus (Figure 1). Over the course of several hours, the estrogen-ER complex then induces a direct response through estrogen response element sequences or an indirect response by triggering expression of other proteins such as transcription factors of the AP1 family, among others. This is viewed as the main 


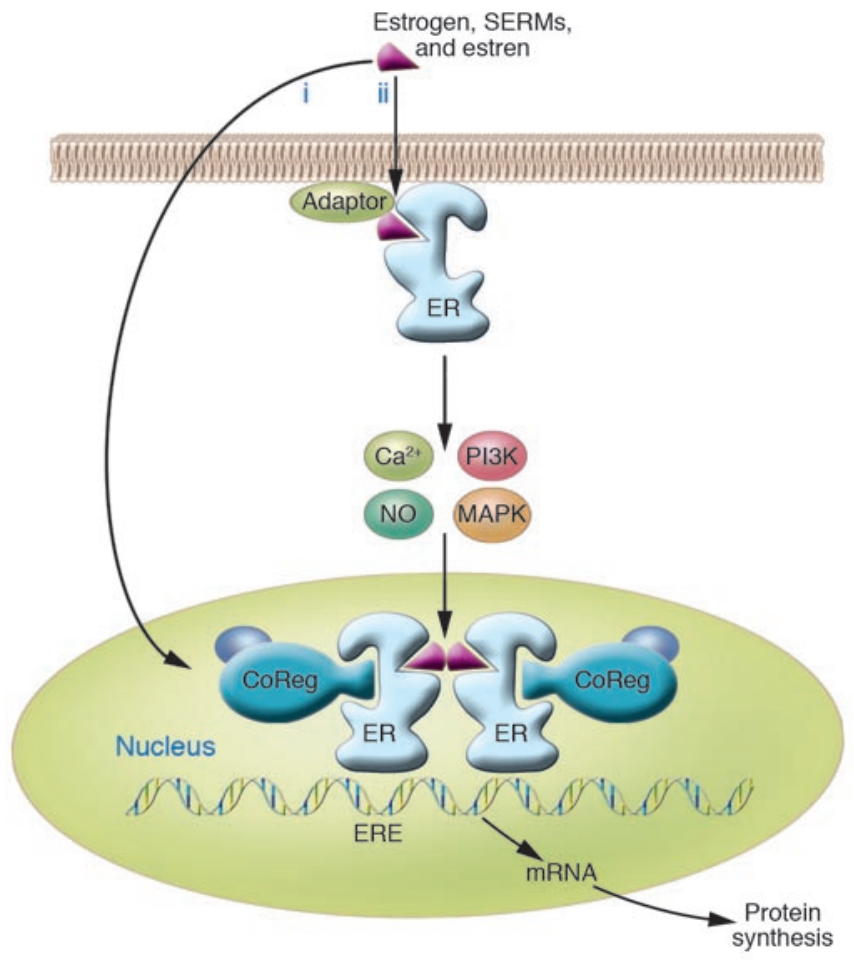

mode of action of estrogen. However, there is also evidence for a rapid, nongenomic response to estrogen. Signaling through the nongenomic pathway can lead to $\mathrm{Ca}^{2+}$ and NO release and activation of various kinases. For a more indepth review of estrogen signaling, see refs. 1 and 2 .

Agonizing and antagonizing the genomic estrogen signaling pathway is complicated by the fact that ERs are expressed in multiple organs. The selective estrogen receptor modulator (SERM) tamoxifen, as an example, activates the ER in bone and uterus but is an antagonist in the breast. Unfortunately, chronic administration of tamoxifen can lead to uterine cancer, so alternative SERMs and other methods for regulating the ER in specific organs have been sought. So far, all described SERMs have been shown to prevent bone loss, but their effects pale in comparison to the anabolic results seen with estrogen and androgen treatment.

\section{Estren provides a solution}

In 2002, Kousteni, Manolagas, and colleagues described a synthetic ligand,

\section{Figure 1}

Pathways of estrogen, SERM, and estren signaling. In the genomic pathway of estrogen action (i), estrogen or SERMs bind to the $E R$, regulating transcription of target genes in the nucleus by binding to estrogen response element (ERE) regulatory sequences and by recruiting coregulatory proteins (CoRegs). Estrens were previously thought only to signal through the rapid, nongenomic pathway mediated by the ER located in or adjacent to the plasma membrane (ii), which may require the presence of adaptor proteins, which target the $\mathrm{ER}$ to the membrane. Activation of the membrane ER leads to a rapid change in cellular signaling molecules and stimulation of kinase activity, which in turn may affect transcription. Figure and legend adapted from ref. 2.

Table 1

Comparison of estren studies

Animal model

Estren source

Estren dose

Age of mice in study

Estren effect on bone

Estren effect on uterus

Estren effect on seminal vesicles

Estren effect on breast cancer cell proliferation
Original estren study (3)

Ovariectomized and orchidectomized Swiss Webster mice

Steraloids Inc., with slow-release pellets from Innovative Research of America

\section{$7.6 \mathrm{mg}$}

6 and 8 months old

Statistically significant increases in BMD and bone strength

None

None 4-estren-3 $\alpha, 17 \beta$-diol (estren), that reproduced the nongenomic effects of estrogen (3). In their hands, estren increased bone mass and strength in gonadectomized Swiss Webster mice but had no effects on uterine or seminal vesicle weight. Estrens also had no effect on the proliferation of MCF-7 human breast cancer cells. The effect of estren was attributed to activation of transcription factors by several kinase cascades (4).

The potent effects of estrens on bone strength suggested that they could be used as a bone anabolic agent in cases of estro-

BMD, bone mineral density.

\section{Current study (7)}

Ovariectomized and orchidectomized

C57BL/6 and ovariectomized Swiss Webster mice

Steraloids Inc. and authors' own synthesized estrens (98\% identical) with slow-release pellets from Innovative Research of America

$7.6 \mathrm{mg}$

3 months old

Preservation of BMD and strength but not anabolic

Statistically significant increase in uterine weight

Statistically significant increase in seminal vesicle weight Increased (on day 6) 
gen deficiency, such as menopause, given that their anabolic effects were restricted to bone. Estrens are currently in preclinical testing; however, the results of their use in humans have not yet been reported.

\section{Upon closer inspection ...}

Since the original description of estren, a few reports have appeared that question whether estren acts in a nongenomic manner (5) and whether it really has no effects on the uterus (6). In the current issue of the JCI, Windahl, Baron, and colleagues report a systematic comparative analysis of estrens in gonadectomized mice to discern whether estrens are in fact nongenomic, bone anabolic compounds with no effects on reproductive organs (7).

The current study (7) reports that while estrens were able to prevent gonadectomyinduced bone loss, they showed no bone anabolic effects when given at the same doses and in the same manner as originally reported (Table 1). Furthermore, and in direct contrast to the original estren study (3), estren was shown to increase uterine and seminal vesicle weight and enhanced the proliferation of human breast cancer cells - the harmful effects estrens were designed to avoid.

Windahl et al. (7) show that estrens bind more strongly to the AR than the ER and suggest that they act more as androgens than estrogens. This was confirmed when the authors treated the gonadectomized animals with estrens and anti-androgens or anti-estrogens, as well as in ER $\alpha-\mathrm{KO}$ mice: the addition of anti-androgen completely blocked the response to estren, and removal of estrogen only partially blocked estren's effects. In agreement with earlier reports (5), estren was shown to have tran- scriptional activity - suggesting that estrens could potentially exert their effects through the genomic pathway in addition to the nongenomic one.

\section{So, who is right?}

There are a few differences in the studies that may lead us to believe that one or the other is more reliable (Table 1). First, the earlier estren study used Swiss Webster mice, while the current authors used C57BL/6 mice (but used Swiss Webster mice when comparing the effects of estrens on uterine weight). Could strain effects account for the differences seen? The real question is whether estrens are bone specific in humans, which remains to be seen.

Also, the age of the animals studied was different - the current study used mice 3-5 months younger than those in the original study, but the authors note that Moverare et al. reported uterotrophic effects of estren in 11-month-old mice at the same doses used here (5). Despite the age difference, the fact that estrens could have an effect on reproductive organs at any stage of life raises serious concerns about their use as a SERM.

Data in the current article (7) show that when the dose of estren was reduced, the adverse effects on reproductive organs disappeared, but, unfortunately, so did the associated bone preservation capacity. Together, the data from the current study and others in the literature make a compelling case that estrens are not suitable for treatment of osteoporosis.

\section{Time for a new SERM}

Given that estren may not be the ideal SERM for treating osteoporosis, the search continues for what could become a blockbuster drug. Some of the authors of the current study have also attempted to enter the fray by testing a new SERM, PSK3471 (7).

The miracle SERM for osteoporosis may be out there somewhere, but it has not been found yet. Perhaps it is PSK3471; perhaps it may still turn out to be estren - results from clinical testing in humans will provide the definitive proof. But until then, the search must continue.

\section{Acknowledgments}

The author wishes to thank members of the bone research community for guidance in preparing this commentary.

Address correspondence to: Ushma S. Neill, Journal of Clinical Investigation, 630 West 168th Street, Box 57A, New York, New York 10032, USA. Phone: (212) 342-0497; Fax: (212) 342-0499; E-mail: editors@the-jci.org.

1. Weitzmann, M.N., and Pacifici, R. 2006. Estrogen deficiency and bone loss: an inflammatory tale. J. Clin. Invest. 116:1186-1194. doi:10.1172/JCI28550.

2. Deroo, B.J., and Korach, K.S. 2006. Estrogen receptors and human disease. J. Clin. Invest. 116:561-570. doi:10.1172/JCI27987.

3. Kousteni, S., et al. 2002. Reversal of bone loss in mice by nongenotropic signaling of sex steroids. Science. 298:843-846.

4. Kousteni, S., et al. 2003. Kinase-mediated regulation of common transcription factors accounts for the bone-protective effects of sex steroids. J. Clin. Invest. 111:1651-1664. doi:10.1172/JCI200317261.

5. Moverare, S., et al. 2003. Estren is a selective estrogen receptor modulator with transcriptional activity. Mol. Pharmacol. 64:1428-1433.

6. Hewitt, S.C., Collins, J., Grissom, S., Hamilton, K., and Korach, K.S. 2006. Estren behaves as a weak estrogen rather than a nongenomic selective activator in the mouse uterus. Endocrinology. 147:2203-2214.

7. Windahl, S.H., et al. 2006. Bone protection by estrens occurs through non-tissue-selective activation of the androgen receptor. J. Clin. Invest. 116:2500-2509. doi:10.1172/JCI28809. 


\title{
Deconstructing endothelial dysfunction: soluble guanylyl cyclase oxidation and the NO resistance syndrome
}

\author{
Mark T. Gladwin
}

Vascular Medicine Branch, National Heart, Lung, and Blood Institute, and Critical Care Medicine Department, Clinical Center, National Institutes of Health, Bethesda, Maryland, USA.

\begin{abstract}
In this issue of the JCI, Stasch and colleagues suggest that a novel drug, BAY 58-2667, potently activates a pool of oxidized and heme-free soluble guanylyl cyclase (sGC; see the related article beginning on page 2552). The increased vasodilatory potency of BAY 58-2667 the authors found in a number of animal models of endothelial dysfunction and in human blood vessels from patients with diabetes suggests that there exists a subphenotype of endothelial dysfunction characterized by receptor-level NO resistance. Diseases associated with NO resistance would appear to be ideally suited for therapies directed at restoring redox homeostasis, sGC activity, and NO sensitivity.
\end{abstract}

Our molecular understanding of the pathogenesis of diabetes mellitus crystallized with the discovery of insulin and the catastrophic failure to produce insulin in type 1 disease. It would take decades to unravel the mechanisms underlying type 2 diabetes, a more common disease associated with preserved insulin production but resistance to insulin at the receptor level. A similar march to discovery characterizes most endocrinopathies; e.g., the identification of a failure to produce thyroid hormone in hypothyroidism and later the discovery of the more unusual generalized resistance to thyroid hormone. If we consider the diatomic free radical NO, a paracrine and endocrine signaling molecule (1), we should not be surprised by the ultimate discovery of "NO resistance syndromes."

Endothelial NO is produced by the endothelial isoform of NOS, eNOS, via a 5-electron oxidation of $\mathrm{L}$-arginine to form L-citrulline and NO. eNOS is activated following stimulation with calcium ionophore, muscarinic receptor activation by acetylcholine, delivery of excess substrate arginine, and shear stress. NO then diffuses as a paracrine signaling molecule

Nonstandard abbreviations used: BAY 58-2667, 4-[((4-carboxybutyl) \{2-[(4-phenethylbenzyl)oxy] phenethyl\}amino) methyl [benzoic]acid; cGMP, cyclic GMP; DEA/NO, donor 2-( $N, N$-diethylamino)-diazenolate-2-oxide; sGC, soluble guanylyl cyclase.

Conflict of interest: The author has declared that no conflict of interest exists.

Citation for this article: J. Clin. Invest. 116:2330-2332 (2006). doi:10.1172/JCI29807. to albuminal smooth muscle and binds to the hemes on the $\alpha / \beta$ heterodimer soluble guanylyl cyclase (sGC), which in turn converts GTP to cyclic GMP (cGMP) and activates cGMP-dependent protein kinases (Figure 1) (2-4). On the heels of the discovery of this pathway, it became clear that patients with coronary artery disease or its risk factors - diabetes, hypercholesterolemia, hypertension, atherosclerosis, increasing age, and tobacco smoking - develop endothelial dysfunction. The observed impairments in stimulated and basal NO production are now classic: The expected blood flow responses to infusion with acetylcholine, an endothelium-dependent vasodilator, are reduced, and the normal decrease in blood flow during NGmonomethyl L-arginine infusion, a direct NOS inhibitor, are blunted (5). However, it is important to note that in all of these pathologies the vasodilatory responses to endothelium-independent exogenous NO, typically assessed by the infusion of sodium nitroprusside, are preserved.

This relatively simple signaling paradigm becomes increasingly complex as we begin to consider factors that modulate substrate transport and availability for NOS, phosphorylation, and posttranslational modifications of NOS; oxidative uncoupling of the enzyme; and downstream stability of the secondary messenger cGMP. Indeed, the enzymes arginase I and II can degrade arginine, and oxidative stress can uncouple eNOS, leading to a state of L-arginine resistance (6-8). Once again, we must note that in all of these pathologies the vasodilatory responses to authentic $\mathrm{NO}$ are generally preserved.

\section{Is there a subphenotype of endothelial dysfunction characterized by NO resistance?}

In this issue of the JCI, Stasch and colleagues present provocative findings that clinical states of endothelial dysfunction can be associated with the accumulation of oxidized and heme-free sGC that cannot be activated by NO (9). Indeed, the oxidation of purified sGC enzyme, endothelial cells, platelets, or aortic ring bioassay preparations with $1 \mathrm{H}-[1,2,4]$ oxadiazolo [3,4-a]quinoxalin-1-one (ODQ) or peroxynitrite produces a state of NO resistance in which both NO-dependent cGMP accumulation and vasodilation are impaired. Remarkably, the authors provide extensive and compelling experimental evidence that the NO- and heme-independent activator of sGC, 4-[((4-carboxybutyl) \{2-[(4phenethylbenzyl)oxy] phenethyl (amino) methyl [benzoic]acid (BAY 58-2667), can potently bind to and activate these oxidized and/or heme-free sGCs, producing selective sGC activation and vasodilation of diseased blood vessels (Figure 1). This binding also appears to inhibit ubiquitindependent sGC protein degradation. The thesis that oxidized and heme-free sGC contributes to endothelial dysfunction in clinical conditions is further supported by in vivo and vascular ring experiments in the spontaneously hypertensive rat, Watanabe hyperlipidemic rabbits, and $\mathrm{ApoE}^{-/-}$mice on a high-fat diet as well as in isolated human mesocolon arteries from patients with type 2 diabetes.

While these studies suggest that atherosclerosis and its risk factors would be associated with impaired sGC function and resistance to exogenous NO, we know that patients with endothelial dysfunction have preserved responses to nitroprusside 